\title{
Phenotypic and Molecular Characterization of Penicillin and Macrolide-Resistant Streptococcus pneumoniae Serotypes Among Pediatric Patients in Addis Ababa, Ethiopia
}

\author{
Abel Abera Negash (iD) ${ }^{1,2}$ \\ Daniel Asrat (iD ${ }^{2}$ \\ Workeabeba Abebe ${ }^{3}$ \\ Abraham Aseffa (1D) \\ Mario Vaneechoutte ${ }^{4}$ \\ 'Armauer Hansen Research Institute \\ (AHRI), Addis Ababa, Ethiopia; \\ ${ }^{2}$ Department of Microbiology, \\ Immunology and Parasitology, School of \\ Medicine, Addis Ababa University, Addis \\ Ababa, Ethiopia; ${ }^{3}$ Department of \\ Pediatrics and Child Health, School of \\ Medicine, Addis Ababa University, Addis \\ Ababa, Ethiopia; ${ }^{4}$ Laboratory \\ Bacteriology Research, Department of \\ Diagnostic Sciences, Faculty of Medicine \\ \& Health Sciences, Ghent University, \\ Ghent, Belgium
}

Background: In several countries, introduction of the pneumococcal conjugate vaccine (PCV) has led to a decline in antimicrobial-resistant pneumococcal disease but has also resulted in a concomitant increase in antimicrobial-resistant, non-vaccine serotypes of Streptococcus pneumoniae. We sought to determine the magnitude of penicillin and macrolide resistance among pneumococcal serotypes and the mechanisms of macrolide resistance in Ethiopia, 5 years after the introduction of PCV10 in the country.

Methods: Susceptibility to penicillin and erythromycin of 119 pneumococcal isolates collected from pediatric patients aged 0-15 years in Addis Ababa, Ethiopia, was tested using disc diffusion, and minimum inhibitory concentration (MIC) was also determined by Etest. Pneumococcal serotypes were determined by sequencing the cpsB gene and using Quellung reaction. Polymerase chain reaction (PCR) and PCR-restriction fragment length polymorphism analysis were used to detect and differentiate the macrolide resistance genes erm(B), mef(A), and mef(B).

Results: Among the 119 isolates, 2.5\% (3/119) were resistant to penicillin, while 58\% (69/119) were intermediate. Resistance to erythromycin was observed in $33.6 \%(40 / 119)$ of the isolates with the highest level of resistance among isolates from middle ear discharge, i.e., 53.3\% (8/15). Half $(19 / 40)$ of the erythromycin resistant isolates were serotype 19A and among serotype 19A isolates, the majority i.e., $54.3 \%$ (19/35) were resistant to erythromycin. The most common macrolide resistance determinant was $m e f(\mathrm{E})$ with a prevalence of $50 \%(20 / 40)$.

Conclusion: Five years after introduction of PCV10 in Ethiopia, we observed that the prevalence of penicillin-resistant $S$. pneumoniae was low. However, there was a high level of macrolide resistance which was mostly in serotype 19A, and the resistance was mainly mediated by efflux pumps. Introduction of PCV13 (which covers serotype 19A) would significantly improve coverage of the macrolide-resistant serotypes. Continued surveillance of pneumococcal serotype distribution and their antibiotic resistance pattern in Ethiopia is warranted.

Keywords: efflux pump, erythromycin, mef(E), PCV10, penicillin, serotype 19A, Streptococcus pneumoniae

\section{Introduction}

Streptococcus pneumoniae (pneumococcus) still remains the major cause of pneumonia, meningitis, bacteremia and otitis media among infants and young children, worldwide. A 2015 estimate indicates that globally, there were 294,000 deaths due to $S$. pneumoniae in children aged 1-59 months. ${ }^{1}$
Correspondence: Abel Abera Negash Armauer Hansen Research Institute (AHRI), P.O. Box 1005, Addis Ababa, Ethiopia

Email abelaberan@gmail.com 
Penicillin non-susceptible pneumococci (PNSP) are on the priority list of antibiotic-resistant bacteria listed by the WHO for which research and development of new antibiotics are required. ${ }^{2}$ PNSP were first detected soon after the discovery of penicillin ${ }^{3}$ and have since spread globally. ${ }^{4}$ A recent meta-analysis of antimicrobial susceptibility profiles for pneumococcal pneumonia in sub-Saharan Africa indicated that the susceptibility to penicillin was $68.6 \%{ }^{5}$

Although the increasing prevalence of macrolideresistant $S$. pneumoniae is raising concerns on the use of macrolides for treating pneumococcal diseases, they still remain an important group of antimicrobials. ${ }^{6}$ In the Ethiopian standard treatment guideline, macrolides are the first alternatives to $\beta$-lactams. ${ }^{7}$ Previous reports from Ethiopia indicate higher resistance to macrolides (23.9\%) compared with other antibiotics $(15.0 \%$ for penicillin and $20.4 \%$ for amoxicillin), in nasopharyngeal pneumococcal isolates $^{8}$ and a macrolide-resistance prevalence of $44.4 \%$ among isolates causing otitis media. ${ }^{9}$

Before the introduction of PCVs, the pneumococcal serotypes with highest level of resistance to penicillin and erythromycin were $6 \mathrm{~B}, 6 \mathrm{~A}, 9 \mathrm{~V}, 14,15 \mathrm{~A}, 19 \mathrm{~F}, 19 \mathrm{~A}$, and $23 \mathrm{~F}$, globally. ${ }^{10}$ After introduction of PCV7 in the vaccination schedule of various countries in the 2000 s and higher valent PCV10 afterwards, there was a significant decline in antimicrobial-resistant pneumococcal carriage and disease due to vaccine serotypes. This effect was however offset by the increasing resistance in non-vaccine serotypes, specifically serotype 19A. ${ }^{11}$

PCV10 was introduced in Ethiopia in October 2011 as a three dose primary series $(3 p+0)$ without any booster dose. ${ }^{12}$ The recent Ethiopian health and demographic survey indicated that $70.1 \%$ children aged $<3$ years surveyed from the whole country and $93.1 \%$ of those from Addis Ababa received all three doses of PCV10. ${ }^{13}$ Most of the previous studies on penicillin resistance in $S$. pneumoniae in Ethiopia ${ }^{8,9}$ used the oxacillin disc diffusion test as a proxy for penicillin resistance and therefore lack accuracy. The CLSI criteria state that for isolates with oxacillin zones $\leq 19 \mathrm{~mm}$, penicillin resistance should not be reported without performing a penicillin MIC test. ${ }^{14}$ In addition, there is a lack of data on the prevalence and distribution of PNSP serotypes in the post-PCV10 era.

In S. pneumoniae, macrolide resistance is mainly mediated by two mechanisms. The first one, target-site modification by erythromycin methylase (erm(B)) gene, usually confers highlevel resistance and cross-resistance to lincosamides and streptogramin $\mathrm{B}$ drugs $\left(\mathrm{MLS}_{\mathrm{B}}\right.$ phenotype). The second mechanism is mediated by mef class of genes that lead to active drug efflux and results in low- to mid-level resistance to macrolides (M phenotype). The mef gene has two variants, mef(A), originally found in Streptococcus pyogenes, and mef (E), which was first described in S. pneumoniae. ${ }^{15}$ The two mef genes have around $90 \%$ sequence homology and can be distinguished using specific primer sets. Due to the sequence similarity between the two genes, they were merged under mef(A) by Roberts and colleagues. ${ }^{16}$ In Ethiopia, in a study performed before the introduction of PCV10, the most common macrolide resistance determinant identified was mef(A/ E). ${ }^{17}$ There are however no data on the magnitude and molecular mechanisms of macrolide resistance among different pneumococcal serotypes in the post-PCV10 era in Ethiopia. Therefore, the aim of this study was to determine the magnitude of penicillin and macrolide resistance among pneumococcal serotypes and the mechanisms of macrolide resistance in Addis Ababa, Ethiopia, 5 years after the introduction of PCV10 in the country.

\section{Materials and Methods Study Sites and Source of Bacterial Isolates}

The pneumococcal isolates were collected from September 2016-August 2017 from children aged 0-15 years attending pediatric emergency departments at Black Lion and Yekatit 12 hospitals and pediatric outpatient departments in Girum hospital and Dr. Yared Pediatric Specialty Center, in Addis Ababa, Ethiopia. A total of 119 S. pneumoniae isolates were collected, comprising 89 from nasopharyngeal swabs of children with community acquired pneumonia, 17 from nasopharyngeal swabs of children with non-respiratory illnesses, 5 from blood of children with pneumonia $(n=1)$ or sepsis $(n=4)$ and 15 from middle ear swabs of children with acute otitis media. Serotypes of most of the pneumococcal isolates (except of the 17 isolates from nasopharyngeal swabs of children with non-respiratory illnesses) were previously determined and have been reported elsewhere. ${ }^{18-21}$

\section{Antimicrobial Susceptibility Testing}

Susceptibility to penicillin was initially determined using the Kirby-Bauer disk diffusion method. ${ }^{22}$ Oxacillin discs (1 $\mu \mathrm{g}$ ) were initially used and for isolates with zones of $\leq$ $19 \mathrm{~mm}$, the minimum inhibitory concentrations (MICs) were determined using Etest strips (bioMérieux, Marcyl'Étoile, France). The results were classified as: susceptible 
$(\leq 0.06 \mu \mathrm{g} / \mathrm{mL})$, intermediate $(0.12-1 \mu \mathrm{g} / \mathrm{mL})$, and resistant $(\geq 2 \mu \mathrm{g} / \mathrm{mL}$ ). To determine macrolide resistance, MICs were determined using Etest strips and results were classified as susceptible (MIC $\leq 0.25 \mu \mathrm{g} / \mathrm{mL}$ ), intermediate $(0.5 \mu \mathrm{g} / \mathrm{mL}$ ), and resistant $(\geq 1 \mu \mathrm{g} / \mathrm{mL}$ ). Test results of both disc diffusion and MICs were interpreted according to the Clinical and Laboratory Standard Institute (CLSI) criteria. ${ }^{14}$ American Type Culture Collection (ATCC) strain S. pneumoniae ATCC 49619 was used for quality control.

\section{DNA Extraction and Serotyping}

DNA from $S$. pneumoniae isolates was extracted using alkaline lysis as previously described. ${ }^{23}$ Initial typing of pneumococcal isolates was done using PCRSeqTyping as previously described ${ }^{24}$ and consisted of amplification and sequencing of a $1061 \mathrm{bp}$ region of the cpsB-gene. Sanger sequencing was performed at GATC Biotech (Constance, Germany) using $20 \mu \mathrm{L}$ of the amplicons. cpsB sequences were then used to interrogate GenBank database (http:// www.ncbi.nlm.nih.gov/blast). BLAST bit score of $>99 \%$ sequence identity with reference sequences was used to assign serotypes. Because the correct serotype could not be assigned by PCRSeqTyping for some of the isolates, serotyping was performed on all isolates as well with the Quellung reaction ${ }^{25}$ using pool and group antisera, obtained from the Statens Serum Institut (Copenhagen, Denmark).

\section{Detection of erm(B) and mef(A/E) Genes}

PCR was used to detect macrolide resistance genes on all macrolide-resistant (MIC $\geq 1 \mu \mathrm{g} / \mathrm{mL}$ ) isolates. Amplification of the ribosomal methylase $(\operatorname{erm}(\mathrm{B}))$ gene was performed using the primers: ermB-F, 5'GAAAAGGTACTAAACCAAATA-3' and 5'-AGTAACG GTACTTAAGTTTAC-3' to generate a 639 bp PCR product. ${ }^{26}$ A PCR-restriction fragment length polymorphism analysis was then performed in order to identify the presence of macrolide efflux genes mef(A/E) and discriminate between $m e f(\mathrm{~A})$ and $m e f(\mathrm{E})$. The primer pair used was: MEF-F, 5'-GCGTTTAAGATAAGCTGGCA-3' and MEF$\mathrm{R}$, 5'-CCTGCACCATTTGCTCCTAC-3', to generate a 1743-bp PCR product. PCR amplification consisted of 35 cycles at $95^{\circ} \mathrm{C}, 54^{\circ} \mathrm{C}$, and $72^{\circ} \mathrm{C}$ for 1 min each. The amplicons were then digested with the BamHI restriction enzyme. $^{27}$

\section{Results}

\section{Susceptibility to Penicillin and Erythromycin}

The most prevalent serotypes in this study were serotypes 19A $(29.4 \%, 35 / 119), 16 \mathrm{~F}(9.2 \%, 11 / 119)$, and nontypeables $(6.7 \%, 8 / 119)$. PCV10 serotypes constituted $11.7 \%(14 / 119)$ whereas PCV13-unique serotypes constituted $32.7 \%$ (39/119). Among the isolates, only $2.5 \%$ (3/ $119)$ were resistant to penicillin, while $58 \%(69 / 119)$ had intermediate susceptibility and 39\% (47/119) were susceptible. The resistant isolates were serotype 19A $(n=2)$ and non typeable $(\mathrm{n}=1)$. Among the isolates with intermediate susceptibility, most, 40.6\% (28/69) were serotype 19A (Figure 1) and among serotype 19A isolates, the majority $(80 \%, 28 / 35)$ showed intermediate susceptibility.

Resistance to erythromycin was observed in $33.6 \%$ (40/119) of the isolates overall. The highest level of erythromycin resistance was seen among isolates from middle ear discharge, $53.3 \%$ (8/15), followed by nasopharyngeal isolates, 32.6\% (29/89), and isolates from blood, 20\% (1/ 5) (Table 1). The resistance level to erythromycin by Etest MIC ranged between 3 and $>256 \mu \mathrm{g} / \mathrm{mL}$, and most $(65.8 \%)$ of the strains showed a high level of resistance $(>256 \mu \mathrm{g} / \mathrm{mL})$. All the three isolates that were resistant to

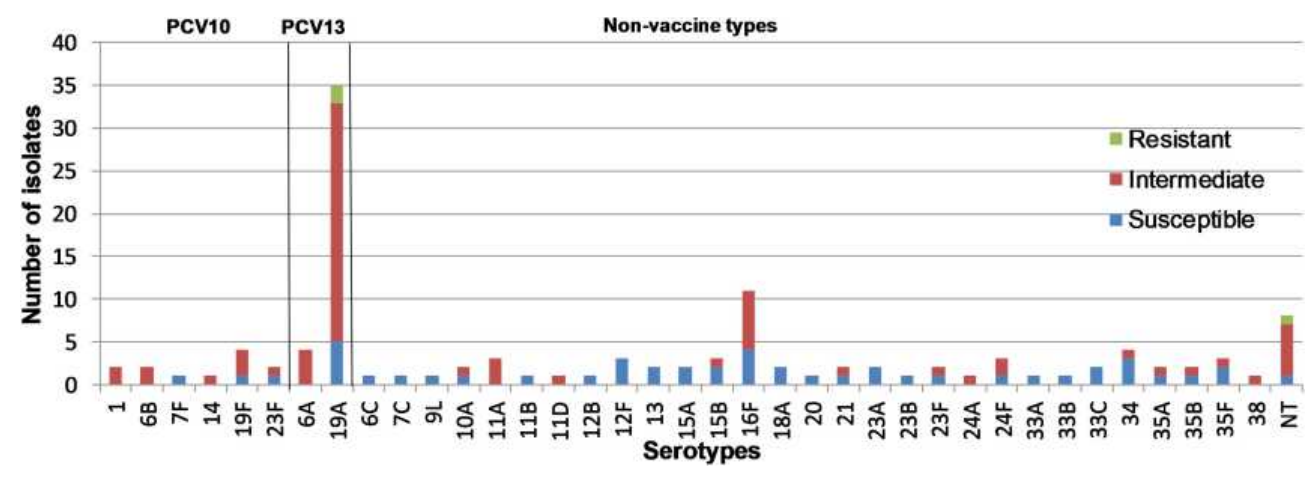

Figure I Pneumococcal serotypes from pediatric patients aged 0-15 years in Addis Ababa, Ethiopia and their susceptibility to penicillin. 
Table I Source Sample, Serotypes and Genotypes of Macrolide-Resistant S. pneumoniae Isolates from Pediatric Patients Aged 0-15 Years in Addis Ababa, Ethiopia

\begin{tabular}{|c|c|c|c|c|c|c|c|c|c|}
\hline \multirow[t]{2}{*}{ Serotype } & \multirow[t]{2}{*}{ Total No. } & \multicolumn{2}{|c|}{$\begin{array}{l}\text { Antimicrobial } \\
\text { Susceptibility }\end{array}$} & \multicolumn{3}{|c|}{ Source Sample } & \multicolumn{3}{|c|}{ Mechanism of Resistance } \\
\hline & & $\mathbf{S}$ & $\mathbf{R}$ & NP & MES & Blood & $\operatorname{erm}(B)$ & $\operatorname{mef}(\mathrm{A})$ & $\operatorname{mef}(\mathrm{E})$ \\
\hline I & 2 & 1 & 1 & & & I & I & & I \\
\hline IIA & 3 & 2 & 1 & 1 & & & & & \\
\hline IID & 1 & & 1 & $\mathrm{I}^{\mathrm{a}}$ & & & & & \\
\hline I2B & I & & 1 & $\mathrm{I}^{\mathrm{a}}$ & & & & & \\
\hline $\mathrm{I} 2 \mathrm{~F}$ & 3 & 2 & 1 & 1 & & & & I & \\
\hline 14 & I & & 1 & 1 & & & & & \\
\hline $15 \mathrm{~A}$ & 2 & I & 1 & 1 & & & I & & \\
\hline I5B & 3 & 2 & 1 & 1 & & & & & I \\
\hline $16 \mathrm{~F}$ & 11 & 8 & 3 & 3 & & & & & 1 \\
\hline 18A & 2 & 1 & 1 & 1 & & & & & I \\
\hline 20 & 2 & I & 1 & 1 & & & & & \\
\hline 19A & 35 & 16 & 19 & 11 & 7 & I & I & & 11 \\
\hline $23 \mathrm{~A}$ & 2 & 1 & 1 & 1 & & & & & I \\
\hline $23 \mathrm{~F}$ & 2 & & 2 & $2^{a}$ & & & & & \\
\hline $35 \mathrm{~A}$ & 2 & 1 & 1 & 1 & & & & & I \\
\hline 38 & I & & 1 & 1 & & & & & I \\
\hline NT & 8 & 5 & 3 & 2 & I & & & & 2 \\
\hline Total No. & 81 & 41 & 40 & 30 & 8 & 2 & 3 & I & 20 \\
\hline
\end{tabular}

Note: ${ }^{a}$ Identified from children with non-respiratory infections.

Abbreviations: S, susceptible; R, resistant; NP, nasopharyngeal swab; MES, middle ear swab.

penicillin were also resistant to erythromycin. Overall, almost half (19/40) of the erythromycin-resistant isolates were serotype 19A and among serotype 19A isolates $54.3 \%(19 / 35)$ were resistant (Figure 2). Among PCV10 serotypes, 6B (2), 7F (1) and 19F (4) were all susceptible to erythromycin, whereas all of serotype 14 (1) and $23 \mathrm{~F}$ (2) isolates were resistant to erythromycin.

\section{Identification of Macrolide Resistance Determinants}

The most common macrolide resistance determinant was mef (E), identified in 50\% (20/40) of the strains while $m e f$ (A) was identified in only one strain $(2.5 \%)$ (Table 1$)$. Among those with mef (E), 55\% (11/20) were serotype 19A. $\operatorname{Erm}(B)$ was identified in three strains, two of which (serotype 1 and 19A) were positive for both $\operatorname{erm}(\mathrm{B})$ and $m e f(\mathrm{E})$ and both had a high level of resistance (MIC > 256). Most of the strains which had $m e f(\mathrm{E}), 65 \%(13 / 20)$ also showed high level of resistance (MIC > 256). In $40 \%$ (16/40) of the resistant erythromycin isolates, neither erm (B) nor $m e f(\mathrm{~A} / \mathrm{E})$ were detected.

\section{Discussion}

Because most antibiotic-resistant pneumococci are serotypes included in PCV, the introduction of PCV has significantly reduced the burden of antibiotic-resistant pneumococcal infections. ${ }^{28}$ In this study, performed 5 years after introduction of PCV10 in Ethiopia, the prevalence of penicillin-non-susceptible (PNSP) isolates was only $2.5 \%$ whereas $58 \%$ were intermediate. In a study on 


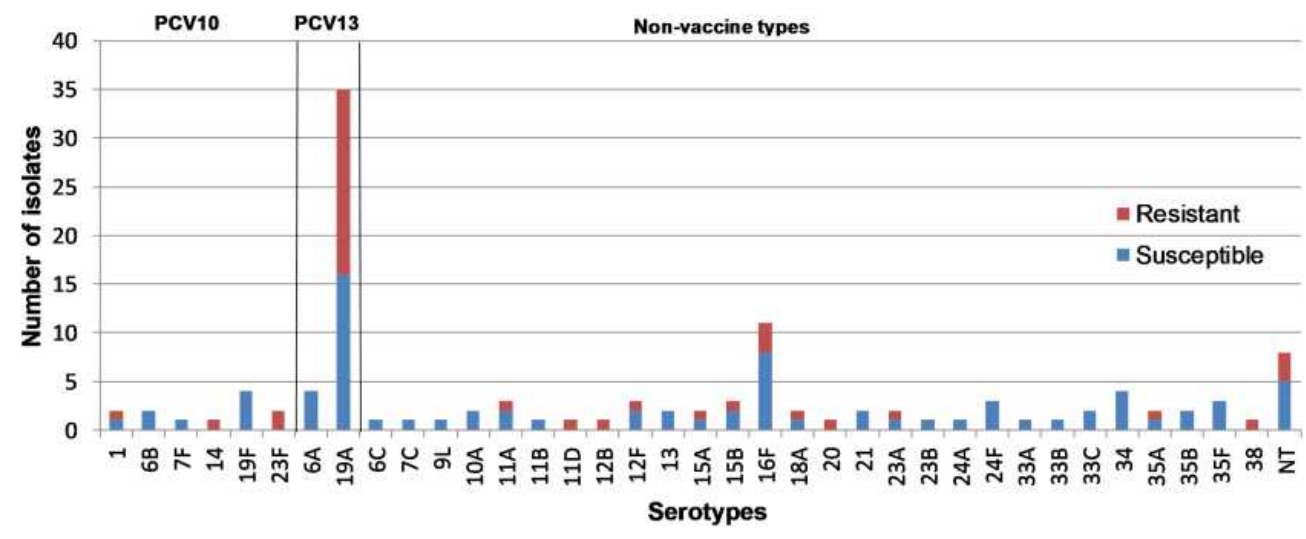

Figure 2 Pneumococcal serotypes from pediatric patients aged 0-15 years in Addis Ababa, Ethiopia and their susceptibility to erythromycin.

pneumococcal isolates from children with meningitis before the introduction of PCV10 in Ethiopia, 17\% (8/ 46) were intermediate and there were no PNSP isolates reported. The isolates with intermediate penicillin MIC levels were serotype 19F $(n=4), 14(n=3)$, and $7(n=$ 1). ${ }^{29}$ In Kenya, a country which introduced PCV10 around the same time as Ethiopia, a similar trend of penicillin susceptibility has been reported. Penicillin susceptibility among pneumococcal isolates from children aged $<5$ years indicated that prevalence of PNSP was low and remained unchanged in the pre-PCV10 period (2009), $2.4 \%$ and post-PCV10 period (2013), $2.7 \%$ while most isolates were intermediate $(77.6 \%$ in 2009 and $74.8 \%$ in 2013). ${ }^{30}$ In most countries that reported a decline in antimicrobial-resistant pneumococcal diseases, the prevalence of antimicrobial resistant vaccine-serotypes was high in the pre-PCV period. $^{11}$ In South Africa for example, 4 years after introduction of PCV7, incidence of invasive pneumococcal disease due to PNSP isolates among children aged $<2$ years of age declined by more than $80 \%{ }^{31}$ In Brazil, PCV10 was introduced in 2010 and analysis of penicillin non-susceptibility among pneumococcal isolates recovered from carriage and invasive disease indicated a decline in the occurrence of PNSP among IPD isolates but an increase among carriage strains. The differences in impact of PCV on the prevalence of PNSP serotypes in different countries and regions indicates that antimicrobial resistance is multifactorial and might be influenced by the different antibiotic use policies and prevalence and nature of serotypes that emerged after introduction of PCV. ${ }^{11,32}$ This also reinforces the need for continued long-term surveillance of the dynamic changes in antimicrobial resistance among pneumococcal isolates due to PCV introduction.
In the current study, two out of the three PNSP isolates and most, $40.6 \%(28 / 69)$ of the penicillin intermediate isolates were serotype 19A and among serotype 19A isolates the majority $(80 \%, 28 / 35)$ were intermediate. In Brazil, after introduction of PCV10, serotype 19A was the most important non-PCV10 serotype that emerged with increased prevalence in both carriage and disease. ${ }^{32}$ In addition, the rate of PNSP isolates among serotype 19A isolates increased significantly $(11.4 \%$ to $64 \%, P<0.001) .{ }^{33}$

Reports from many parts of the world indicate that macrolide-resistant $S$. pneumoniae are now more common than PNSP. ${ }^{34}$ In the current study, $33.6 \%$ of the pneumococcal isolates were resistant to erythromycin out of which half were serotype 19A. Our findings were similar to a study on antimicrobial resistance among nasopharyngeal pneumococcal isolates from pediatric outpatients in Northern Ethiopia (33.2\%) performed after the introduction of PCV10. ${ }^{35}$ In a study performed before the introduction of PCV10 in Ethiopia on the distribution of nasopharyngeal pneumococcal serotypes before and after mass azithromycin treatment for trachoma in Northern Ethiopia, the prevalence of pneumococcal serotype 19A was quite low $(2 \%) .{ }^{36}$ In the same study, the resistance level for azithromycin increased from $0 \%$ pre-treatment to $50 \%$ post-treatment as it did for most other serotypes, indicating the impact of treatment on selection of antibiotic resistant strains. In Brazil, prevalence of erythromycin resistance among serotype 19A isolates from children aged $<5$ years of age increased from $20.5 \%$ pre-PCV10 period to $85.7 \%$ in $6-7$ years after PCV10 introduction and factors such as the pressure of vaccine and antimicrobial use and introduction and spread of multidrug resistant clones have been cited as possible reasons for this increase. ${ }^{33}$ In Ethiopia, although there is a lack of surveillance data on 
antimicrobial resistance among different pneumococcal serotypes, the high prevalence of macrolide resistance especially among serotype 19A in this study may be due to the pressure of PCV10 and the use of antibiotics.

Results from a randomized double-blind trial that assessed the impact of PCV13 over PCV7 on reducing carriage of antibiotic-resistant pneumococci in Israel indicated that PCV13 significantly reduced the acquisition of pneumococci non-susceptible to penicillin, erythromycin, and clindamycin, mainly seen in serotypes $19 \mathrm{~F}, 6 \mathrm{~A}$, and $19 \mathrm{~A} .{ }^{37}$ It is also hoped that the introduction of PCV13 in Ethiopia will help to reduce acquisition of antibioticresistant pneumococci, especially serotype 19A.

In the current study, the most common macrolide resistance mechanism was macrolide efflux, identified in $55 \%$ of the macrolide resistant isolates. The results were similar to the only previous study in Ethiopia that identified macrolide resistance mechanisms. ${ }^{17}$ In that study, performed before the introduction of PCV10 in Ethiopia, discrimination was not made between $m e f(\mathrm{~A})$ and $m e f(\mathrm{E})$ genes. In the current study, macrolide efflux was the main macrolide resistance mechanism and $m e f(\mathrm{E})$ was the most common gene. Previous findings indicate that $m e f(\mathrm{E})$ is predominant in the United States, South Africa, and Asia while $m e f(\mathrm{~A})$ is more common in Europe.$^{38}$ Although macrolide resistance encoded by mef class of genes in $S$. pneumoniae is thought to confer low- to mid-level resistance, ${ }^{15}$ in our study, $65 \%$ of the strains with mef(E) had high level of resistance (MIC $>256 \mu \mathrm{g} / \mathrm{mL}$ ). A recent study has indicated the ability of $S$. pneumoniae to generate high-level macrolide resistance by macrolide efflux and in the presence of macrolides, thus offering a fitness advantage to S. pneumoniae. ${ }^{39}$

In $40 \%(16 / 40)$ of the macrolide-resistant isolates, neither $\operatorname{erm}(\mathrm{B})$ nor $m e f(\mathrm{~A} / \mathrm{E})$ were detected. This indicates that other less common macrolide resistance mechanisms such as methyltransferase genes $\operatorname{erm}(\mathrm{A})$ and $\operatorname{erm}(\mathrm{C})$, the efflux pump gene mef(I), or mutations in $23 \mathrm{~S}$ rRNA, L4, and L22 proteins might be responsible, ${ }^{40}$ which requires further investigation.

We acknowledge that our study had some limitations. First, the number of isolates is relatively small and more isolates would have been useful. Second, the focus of our study was on the most common macrolide resistance mechanisms and we did not test the presence of the less common macrolide-resistance mechanisms. Despite these limitations, however, to the best of our knowledge, this is the first report of the distribution of penicillin- and macrolide-resistant pneumococcal serotypes and the prevalence of macrolide-resistant pneumococcal genotypes in the post-PCV10 era in Ethiopia.

\section{Conclusions}

Findings from the study indicate that penicillin resistance among $S$. pneumoniae isolates, 5 years after the introduction of PCV10 in Ethiopia, is low, with most PNSP isolates being intermediately susceptible. Resistance to macrolides on the other hand was high and half of the isolates of the most prevalent serotype 19A were resistant to macrolides. Among macrolide-resistant isolates, the most common mechanism of resistance was macrolide efflux, with predominance of mef(E). Continued studies on the impact of antibiotic treatment and $\mathrm{PCV}$ on the prevalence and distribution of antibiotic-resistant pneumococcal serotypes in Ethiopia are warranted and introduction of PCV13 would significantly enhance the coverage of the predominantly macrolide-resistant serotype 19A.

\section{Ethics Approval and Consent to Participate}

The study procedures were in accordance with the Helsinki Declaration. The study was approved by the AHRI/All Africa Leprosy Rehabilitation and Training Hospital (ALERT) Ethics Review Committee, Addis Ababa University Institutional Review Board, Yekatit 12 Medical College Ethics Committee and the National Research Ethics Review Committee (No. 310/194/2017). The parents and/or guardians of all participants gave written informed consent.

\section{Acknowledgments}

We wish to express our sincere gratitude to all children and guardians who participated in the study, and to the physicians and nurses who participated in patient recruitment and sample collection.

\section{Author Contributions}

All authors made substantial contributions to conception and design, acquisition of data, or analysis and interpretation of data; took part in drafting the article or revising it critically for important intellectual content; agreed to submit to the current journal; gave final approval of the version to be published; and agree to be accountable for all aspects of the work.

\section{Funding}

This research has been supported by a $\mathrm{PhD}$ Scholarship for AAN from the Belgian Development Cooperation through 
VLIR-UOS. The funder had no role in study design, data collection, analysis, interpretation and writing the manuscript.

\section{Disclosure}

Dr Abel Abera Negash reported grants from VLIR-UOS during the conduct of the study. The authors reported no other potential conflicts of interest for this work.

\section{References}

1. Wahl B, O'Brien KL, Greenbaum A, et al. Burden of Streptococcus pneumoniae and Haemophilus influenzae type $\mathrm{b}$ disease in children in the era of conjugate vaccines: global, regional, and national estimates for 2000-15. Lancet Glob Health. 2018;6(7):e744-e757. doi:10.1016/S2214-109X(18)30247-X

2. World Health Organization. Global Priority List of AntibioticResistant Bacteria to Guide Research, Discovery, and Development of New Antibiotics. Vol. 43. Geneva, Switzerland: World Health Organization; 2013.

3. Chiou CC. Does penicillin remain the drug of choice for pneumococcal pneumonia in view of emerging in vitro resistance? Clin Infect Dis. 2006;42(2):234-237. doi:10.1086/499596

4. Klugman KP. Pneumococcal resistance to antibiotics. Clin Microbiol Rev. 1990;3(2):171-196. doi:10.1128/CMR.3.2.171

5. Iroh Tam PY, Sadoh AE, Obaro SK. A meta-analysis of antimicrobial susceptibility profiles for pneumococcal pneumonia in Sub-Saharan Africa. Paediatr Int Child Health. 2018;38(1):7-15. doi:10.1080/ 20469047.2017.1298700

6. Cheng AC, Jenney AWJ. Macrolide resistance in pneumococci-is it relevant? Pneumonia. 2016;8(1):10. doi:10.1186/s41479-016-0010-1

7. FMHACA. Standard treatment guidelines for general hospitals. Addis Ababa, Ethiopia: Food, Medicine and Health Care Administration and Control Authority; 2014. Available from: http:// www.fmhaca.gov.et/wp-content/uploads/2019/03/STG-GeneralHospital.pdf. Accessed January 9, 2021.

8. Abaye G, Fekadu H, Haji K, Alemu D, Anjulo AA, Yadate DT. Prevalence and risk factors of pneumococcal nasopharyngeal carriage in healthy children attending kindergarten, in district of Arsi Zone, South East, Ethiopia. BMC Res Notes. 2019;12(1):253. doi:10.1186/ s13104-019-4283-3

9. Hailegiyorgis TT, Sarhie WD, Workie HM. Isolation and antimicrobial drug susceptibility pattern of bacterial pathogens from pediatric patients with otitis media in selected health institutions, Addis Ababa, Ethiopia: a Prospective Cross-Sectional Study. BMC Ear Nose Throat Disord. 2018;18(1):8. doi:10.1186/s12901-018-0056-1

10. Liñares J, Ardanuy C, Pallares R, Fenoll A. Changes in antimicrobial resistance, serotypes and genotypes in Streptococcus pneumoniae over a 30-year period. Clin Microbiol Infect. 2010;16(5):402-410. doi:10.1111/j.1469-0691.2010.03182.x

11. Tin Tin Htar M, van Den Biggelaar AHJ, Sings H, et al. The impact of routine childhood immunization with higher-valent pneumococcal conjugate vaccines on antimicrobial-resistant pneumococcal diseases and carriage: a systematic literature review. Expert Rev Vaccines. 2019;18(10):1069-1089. doi:10.1080/14760584.2019.1676155

12. FMOH. Introducing pneumococcal conjugate vaccine in Ethiopia: training manual for health workers. Addis Ababa, Ethiopia: Federal Democratic Republic of Ethiopia, Ministry of Health; 2011. Avialable from: https://www.medbox.org/pdf/5e148832db60a2044c2d3dd9. Accessed January 10, 2021.

13. Ethiopian Public Health Institute (EPHI) and ICF. Ethiopia mini demographic and health survey 2019: key indicators. EPHI and ICF; 2019. Avialable from: https://dhsprogram.com/pubs/pdf/ PR120/PR120.pdf. Accessed April 8, 2021.
14. CLSI. Performance Standards for Antimicrobial Susceptibility Testing. Twenty Eighth Informational Supplement M100-S27. Wayne, PA: Clinical and Laboratory Standards Institute; 2017.

15. Rudolph K, Bulkow L, Bruce M, et al. Molecular resistance mechanisms of macrolide-resistant invasive Streptococcus pneumoniae isolates from Alaska, 1986 to 2010. Antimicrob Agents Chemother. 2013;57(11):5415-5422. doi:10.1128/AAC.00319-13

16. Roberts MC, Sutcliffe J, Courvalin P, Jensen LB, Rood J, Seppala H. Nomenclature for macrolide and macrolide-lincosamidestreptogramin B resistance determinants. Antimicrob Agents Chemother. 1999;43(12):2823-2830. doi:10.1128/aac.43.12.2823

17. Skalet AH, Cevallos V, Ayele B, et al. Antibiotic selection pressure and macrolide resistance in nasopharyngeal Streptococcus pneumoniae: a cluster-randomized clinical trial. PLoS Med. 2010;7(12): e1000377. doi:10.1371/journal.pmed.1000377

18. Negash AA, Asrat D, Abebe W, et al. Bacteremic community-acquired pneumonia in Ethiopian children: etiology, antibiotic resistance, risk factors, and clinical outcome. Open Forum Infect Dis. 2019;6(3):ofz029. doi:10.1093/ofid/ofz029

19. Negash AA, Asrat D, Abebe W, et al. Pneumococcal carriage, serotype distribution, and risk factors in children with community-acquired pneumonia, 5 years after introduction of the 10 -valent pneumococcal conjugate vaccine in Ethiopia. Open Forum Infect Dis. 2019;6(6):ofz259. doi:10.1093/ofid/ofz259

20. Negash AA, Asrat D, Abebe W, et al. Pneumococcal serotype 19A is the major cause of pediatric acute otitis media with ruptured tympanic membrane in Addis Ababa, Ethiopia, 5 years after the introduction of the ten-valent pneumococcal conjugate vaccine. Int $J$ Pediatr Otorhinolaryngol. 2019;126:109638. doi:10.1016/j.jpporl.2019.109638

21. Negash AA, Asrat D, Abebe W, et al. Etiology, antibiotic susceptibility and prognostic factors of pediatric community-acquired sepsis in Addis Ababa, Ethiopia. J Infect Dev Ctries. 2021;15(01):113-122. doi:10.21203/rs.3.rs-30916/v1

22. Bauer AW, Kirby WM, Sherris JC, Turck M. Antibiotic susceptibility testing by a standardized single disk method. Am J Clin Pathol. 1966;45(4):493-496. doi:10.1093/ajcp/45.4_ts.493

23. Vaneechoutte M, Claeys G, Steyaert S, et al. Isolation of Moraxella canis from an ulcerated metastatic lymph node. J Clin Microbiol. 2000;38(10):3870-3872. doi:10.1128/JCM.38.10.3870-3871.2000

24. Nagaraj G, Ganaie F, Govindan V, Ravikumar KL. Development of PCRSeqTyping - a novel molecular assay for typing of Streptococcus pneumoniae. Pneumonia. 2017;9(1):8-17. doi:10.1186/s41479-0170032-3

25. Sørensen UBS. Typing of pneumococci by using 12 pooled antisera. $J \quad$ Clin Microbiol. 1993;31(8):2097-2100. doi:10.1128/ JCM.31.8.2097-2100.1993

26. Sutcliffe J, Grebe T, Tait-Kamradt A, Wondrack L. Detection of erythromycin-resistant determinants by PCR. Antimicrob Agents Chemother. 1996;40(11):2562-2566. doi:10.1128/aac.40.11.2562

27. Tait-Kamradt A, Clancy J, Cronan M, et al. mefE is necessary for the erythromycin-resistant M phenotype in Streptococcus pneumoniae. Antimicrob Agents Chemother. 1997;41(10):2251-2255. doi:10.1128/AAC.41.10.2251

28. Ginsburg AS, Klugman KP. Vaccination to reduce antimicrobial resistance. Lancet Glob Health. 2017;5(12):e1176-e1177. doi:10.1016/S2214-109X(17)30364-9

29. Muhe L, Klugman KP. Pneumococcal and Haemophilus influenzae meningitis in a children's hospital in Ethiopia: serotypes and susceptibility patterns. Trop Med Int Health. 1999;4(6):421-427. doi:10.1046/j.1365-3156.1999.00417.x

30. Kobayashi M, Bigogo G, Kim L, et al. Impact of 10-valent pneumococcal conjugate vaccine introduction on pneumococcal carriage and antibiotic susceptibility patterns among children aged $<5$ years and adults with human immunodeficiency virus infection: Kenya, 2009-2013. Clin Infect Dis. 2020;70(5):814-826. doi:10.1093/cid/ ciz 285 
31. von Gottberg A, de Gouveia L, Tempia S, et al. Effects of vaccination on invasive pneumococcal disease in South Africa. $N$ Engl $J$ Med. 2014;371(20):1889-1899. doi:10.1056/nejmoa1401914

32. Pinto TCA, Neves FPG, Souza ARV, et al. Evolution of penicillin non-susceptibility among Streptococcus pneumoniae isolates recovered from asymptomatic carriage and invasive disease over 25 years in Brazil, 1990-2014. Front Microbiol. 2019;10(MAR):1-10. doi:10.3389/fmicb.2019.00486

33. Cassiolato AP, Grassi Almeida SC, Andrade AL, Minamisava R, Cristina de Cunto Brandileone M. Expansion of the multidrug-resistant clonal complex 320 among invasive Streptococcus pneumoniae serotype 19A after the introduction of a ten-valent pneumococcal conjugate vaccine in Brazil. PLoS One. 2018;13(11):e0208211. doi:10.1371/journal.pone.0208211

34. Farrell DJ, Morrissey I, Bakker S, Felmingham D. Molecular characterization of macrolide resistance mechanisms among Streptococcus pneumoniae and Streptococcus pyogenes isolated from the PROTEKT 1999-2000 study. J Antimicrob Chemother. 2002;50(SUPPL.1):39-47. doi:10.1093/jac/dkf806

35. Assefa A, Gelaw B, Shiferaw Y, Tigabu Z. Nasopharyngeal carriage and antimicrobial susceptibility pattern of Streptococcus pneumoniae among pediatric outpatients at Gondar university hospital, North West Ethiopia. Pediatr Neonatol. 2013;54(5):315-321. doi:10.1016/ j.pedneo.2013.03.017
36. Keenan JD, Sahlu I, McGee L, et al. Nasopharyngeal pneumococcal serotypes before and after mass azithromycin distributions for trachoma. $J$ Pediatric Infect Dis Soc. 2016;5(2):223-226. doi:10.1093/jpids/piu143

37. Dagan R, Juergens C, Trammel J, et al. Efficacy of 13-valent pneumococcal conjugate vaccine (PCV13) versus that of 7-valent PCV (PCV7) against nasopharyngeal colonization of antibiotic-nonsusceptible Streptococcus pneumoniae. J Infect Dis. 2015;211(7):1144-1153. doi:10.1093/infdis/jiu576

38. Daly MM, Doktor S, Flamm R, Shortridge D. Characterization and prevalence of MefA, MefE, and the associated $\operatorname{msr}(\mathrm{D})$ gene in Streptococcus pneumoniae clinical isolates. J Clin Microbiol. 2004;42(8):3570-3574. doi:10.1128/JCM.42.8.3570-3574.2004

39. Schroeder MR, Lohsen S, Chancey ST, Stephens DS. High-level macrolide resistance due to the mega element $[\mathrm{mef}(\mathrm{E}) / \mathrm{mel}]$ in Streptococcus pneumoniae. Front Microbiol. 2019;10:868. doi:10.3389/fmicb.2019.00868

40. Schroeder MR, Stephens DS. Macrolide resistance in Streptococcus pneumoniae. Front Cell Infect Microbiol. 2016;6:98. doi:10.3389/ fcimb.2016.00098

\section{Publish your work in this journal}

Infection and Drug Resistance is an international, peer-reviewed openaccess journal that focuses on the optimal treatment of infection (bacterial, fungal and viral) and the development and institution of preventive strategies to minimize the development and spread of resistance. The journal is specifically concerned with the epidemiology of antibiotic resistance and the mechanisms of resistance development and diffusion in both hospitals and the community. The manuscript management system is completely online and includes a very quick and fair peerreview system, which is all easy to use. Visit http://www.dovepress.com/ testimonials.php to read real quotes from published authors. 